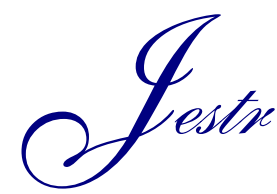

Research Article

\title{
Automatic Segmentation and Classification of White Blood Cells in Peripheral Blood Samples
}

\author{
Ali Mohammad Alqudah ${ }^{1, *}$, Ola Al-Ta'ani ${ }^{2}$ and Alaa Al-Badarneh ${ }^{1}$ \\ ${ }^{1}$ Department of Biomedical Systems and Informatics Engineering, Yarmouk University, Irbid, Jordan. \\ ${ }^{2}$ Department of Computer Engineering, Yarmouk University, Irbid, Jordan.
}

Received 5 May 2018; Accepted 24 December 2018

\begin{abstract}
White blood cells analysis is generally performed for helping specialists in evaluating a wide range of hematic pathologies such as acquired immune deficiency syndrome (AIDS), blood cancer (leukemia) and other related diseases. Segmentation, Counting, and classification of leukocytes or white blood cells (WBC) in the peripheral blood samples images provide informative data about the samples. Therefore, performing them in the most efficient way is very important in the hematological analysis procedure. Unfortunately, the traditional manual segmentation method is very tedious, time-consuming and provides inaccurate results due to the human factor. Hence, a computer-aided segmentation and classification system is needed to make the process both fast and accurate. In this paper, a new and completely automated system for different types of WBCs segmentation is proposed. The system is evaluated on peripheral blood smears from whole slide images based on color space transformation. In the segmentation process of the WBC, image enhancement techniques were applied on saturation frame of HSV color space and a simple thresholding technique was used to find the brightest object. Moreover, three types of features were extracted from the segmented WBCs which are morphological, statistical, and textural. Order of features is performed using the principal component analysis (PCA). Then, the performance of three classifiers probabilistic neural network (PNN) and support vector machine (SVM) and Random Forest Tree is obtained. In total, the results show that the proposed method is accurate and sufficient to be applied in hematological laboratories. The average accuracy of segmentation was $98.98 \%$ and classification was $99.6 \%$.
\end{abstract}

Keywords: Leukocytes, white blood cells, peripheral blood, segmentation, classification, PNN, SVM, and random forest.

\section{Introduction}

Blood smear digital images from microscopes provide important information about the blood components that can be used by blood hematology specialists for diagnosis and disease prediction purposes. Usually, blood samples are prepared and sent to machines called blood cells counters in order to count the number of different cells types. If an abnormal number of cells is found, the blood smear microscopic image is rechecked, and the cause of the abnormality based on the morphological properties of the cells is investigated. Visual inspection of the blood cells is time consuming, inaccurate and tedious $[1,2,3]$.

There are three main types of blood cells: red blood cells (RBCs), white blood cells (WBCs), and platelets. RBCs are responsible for oxygen and carbon mono oxide content transportation from and toward all living organism inside the human body. They normally form up to $40-50 \%$ of the total blood volume with a diameter of 6-8 $\mu \mathrm{m}$. Platelets are a disc like shape with dimension of $1 \times 4 \mu \mathrm{m}$ and play a main functionality in the vascular clotting process or hemostasis. They form around $0.1 \%$ of the total blood volume [3,4,5].

WBCs are a major component of the body's immune system which is responsible for defending against foreign invaders and infectious diseases. WBCs, in general, are classified into two main categories: granulocytes and agranulocytes. They can be further divided into five subtypes. Among Granulocytes, there are basophil, eosinophil, and neutrophil. While lymphocyte and monocytes are among agranulocytes [6,7]. More details about the WBC types are shown in Table 1.

Table 1. Types of WBCs with information about approximated diameter and percentage in adults [7,21]

\begin{tabular}{|c|c|c|c|c|c|}
\hline \multirow{2}{*}{ Type } & \multicolumn{3}{|c|}{ Granulocytes } & \multicolumn{2}{|c|}{ Agranulocytes } \\
\hline & $\begin{array}{c}\text { Basop } \\
\text { hil }\end{array}$ & $\begin{array}{l}\text { Eosin } \\
\text { ophil }\end{array}$ & $\begin{array}{l}\text { Neutr } \\
\text { ophil }\end{array}$ & $\begin{array}{c}\text { Lymph } \\
\text { ocyte }\end{array}$ & $\begin{array}{l}\text { Mono } \\
\text { cyte }\end{array}$ \\
\hline $\begin{array}{l}\text { Micro- } \\
\text { scopic } \\
\text { Image }\end{array}$ & & & & & \\
\hline $\begin{array}{l}\text { Expre- } \\
\text { ssive } \\
\text { Image }\end{array}$ & 210 & $(x)$ & 8 & & \\
\hline $\begin{array}{c}\text { Perce- } \\
\text { ntage in } \\
\text { adults } \%\end{array}$ & 0.4 & 2.3 & 62 & 30 & 5.2 \\
\hline $\begin{array}{l}\text { Diameter } \\
\mu \mathrm{m}\end{array}$ & $10-16$ & $9-15$ & $9-15$ & $\begin{array}{c}\text { Small } \\
7-8 \\
\text { Large } \\
12-18\end{array}$ & $12-20$ \\
\hline
\end{tabular}


Segmentation and type recognition of WBCs in the peripheral blood samples images can assist the hematology specialist in diagnosis the diseases like AIDS, leukemia and blood cancer, which make this process the most important step in the hematological analysis procedure [8]. The process of segmentation and type recognition can be done using automatics or manual process. The manual process is done by searching for the cells and determining their type, the process totally based on the human and has a high risk of miss-recognition of the types due to human factors [9]. Automatic methods are usually done using special instruments such as flow cytometers, automatic cell counter and recognition machines which are based on the coulter method, laser methods or modified version of them [10, 11]. Automatic counting and recognition machines are very expensive due to their cutting-edge technology and they provide a piece of quantitative and not qualitative information about the WBC $[12,13]$.

Due to recent advancements in the medical image processing and analysis and artificial intelligence algorithms, many methods have been proposed for detection, segmentation, and recognition of WBCs. Such methods provide quantitate and qualitative information about each type of WBC $[14,15]$. The systems that combine image processing-artificial intelligence system can help hematology specialists with high accurate segmentation and information about WBC [16,17].

Among detection, segmentation, and recognition of WBCs, Segmentation is the most challenging part. In the literature, many methods have been proposed for WBCs segmentation which can be divided into three main categories: color space transformations which is the most common one, morphology-based methods, and hybrid methods. For color space transformation methods, Li et al. proposed a dual-threshold method that was applied to a combination of RGB and HSV color space, the segmentation technique attained an accuracy of $97.85 \%$. While Ramesh etc. al. proposed a method that combines color transformation and morphological operations and an accuracy of $93.1 \%$ was achieved. Then a two-step classification was applied with an overall accuracy of $93.9 \%$ [20].

For the morphological based methods, Prinyakupt and Pluempitiwiriyawej introduced a fast, robust, and efficient method that implements the ellipse fitting technique to localize the WBCs. They used a set of morphological features to describe cells and nucleus properties and applied linear and naïve Bayes classifiers to recognize them [18]. While Gautam and Bhadauria proposed two methods for segmentation using combination of morphological operations one for training and the other for testing, then they applied different types of classifiers based on structural features (area, perimeter, eccentricity, and circularity) with total accuracy of $73 \%$ (highest: $100 \%$ for lymphocytes, lowest: $50 \%$ for monocyte) [21].

In hybrid methods, Liu etc. al. suggested a combined method between nucleus mark watershed operations and mean shift clustering for segmentation of WBCs [19], while Ravikumar proposed a novel technique for WBCs segmentation based on fast-RVM with an overall accuracy of $92.4 \%$ [14]. Shirazi etc. al. considered a combination of Wiener filter with Curvelet transform for image enhancement and denoising. In other words, they combined the two methods, entropy filter and mathematical morphology for image segmentation. In the classification process, they used geometrical, intensity and textural features as features set. The method was efficient for segmentation and classification and the accuracy of each type of WBC were $100 \%, 96.15 \%, 92.30 \%, 92.30 \%$ and $96.15 \%$ for basophil, eosinophil, monocyte, lymphocyte, and neutrophil respectively [22]. Sajjad etc al. proposed a framework for WBCs segmentation then classification multi-class ensemble classification using SVM based on texture, statistical, and wavelet features selected by principal component analysis (PCA). The overall accuracy of the segmentation process was $94.7 \%$ while the overall accuracy of the classifier was $98.6 \%$ [23].

In this paper, a new method for segmentation and classification of WBCs based on the color transformation is proposed. The method combines the color transformation with image morphological methods. A set of features are extracted to classify the segmented WBCs. The features include texture, statistical and morphological features. After that, the features are fed into PCA to order them based on their contribution of distinguishing between the different WBC types. Finally, the selected features are fed into PNN, SVM, and random forest classifiers.

The rest of this paper is organized as follows. In Section 2 , the methodology architecture and implementation are discussed. The methods used for automatic recognition of five types of white blood cells in hematological images are provided in Section 3. In Section 4, results are presented and discussed. Finally, the paper is concluded in Section 5.

\section{Methodology}

In the peripheral blood of a healthy human, the average diameter of platelets, RBCs and WBCs are $2-3 \mu \mathrm{m}, 6-8 \mu \mathrm{m}$ and $8-20 \mu \mathrm{m}$ respectively. Due to the variation in the size of the blood components, the WBC alone can be segmented, and the output can be classified into only five WBC types. The overall automated approach for cells segmentation and classification in the peripheral blood samples mainly consists of four steps illustrated in Figure 1. The approach includes input image, cells segmentation methodology, feature extraction, and classification.

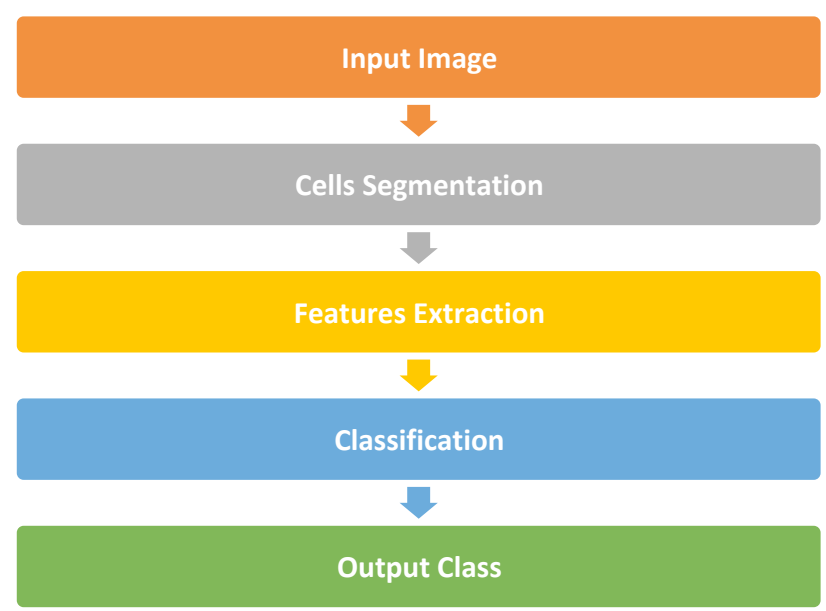

Fig. 1. The general structure of WBC segmentation and classification systems.

Designing an automated method for highly accurate WBC segmentation and classification is the main aim of this paper. In order to segment the WBC, a four main parts method is proposed. The method includes image enhancements, cells segmentation, feature extraction, and classification. The 
detailed structure of the proposed method is shown in Figure 2 and each step will be discussed thoroughly in the following sections.

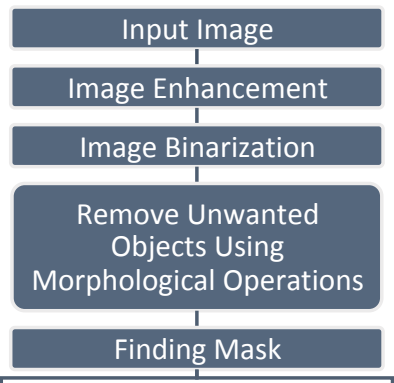

\begin{tabular}{c|c|c|}
\hline Masking Original Image & Finding Bounding Box \\
& $\begin{array}{c}\text { Applying Bounding Box on } \\
\text { Original Image and Crop } \\
\text { Cell(s) Area }\end{array}$ \\
\hline
\end{tabular}

Final Croped Masked Image

Fig. 2. The structure of the proposed WBC segmentation system. 2.1. Image Enhancement

Image enhancements are mandatory [2,5]. Otherwise, miss segmentation cells would appear and classification accuracy would be low. The image enhancement procedure in our proposed method mainly depends on color space transformation and spatial domain adjustment and filtration. Figure 3 shows the image enhancement procedure.

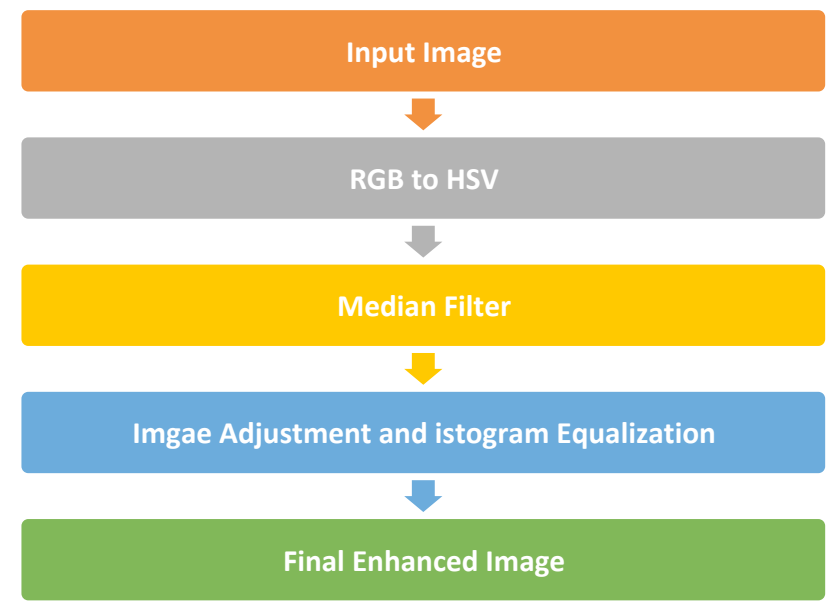

Fig. 3. Enhancement procedure of peripheral blood sample image.

Our proposed approach starts with converting the image from RGB (Red, Green and Blue frames) color space to HSV (Hue, Saturation and Value frames) color space. HSV color space focuses on the visual perception of the variation in Hue, Saturation, and Intensity values of an image pixel. Also, it describes either the color value or hue in terms of two other components: saturation and brightness value. Pixel features were extracted by choosing one of the three frames as the dominant property $[3,4,9]$.

Comparing all three frames of the microscopic image in the RGB and HSV color spaces (shown in Figure 4), the $\mathrm{WBCs}$ are clearly visible and easy to be segmented in the saturation frame. Hence, it is the one been adopted in our approach.

After that, a median filter is applied in order to reduce the noise in the saturation frame. The median filter would cause some blurring in the edge of detected cells as shown in Figure $5 \mathrm{~A}$. Then, image adjusting followed by histogram equalization is applied to the output image of the median filter. Image adjusting would increase the contrast resolution of the image by saturating the bottom $1 \%$ and the top $1 \%$ of all pixel values as shown in Figure $5 \mathrm{~B}$. While, the histogram equalization would enhance the contrast by mapping each pixel intensity value to a new one based on the histogram of the image. The final output image of this procedure is shown in Figure $5 \mathrm{C}$.
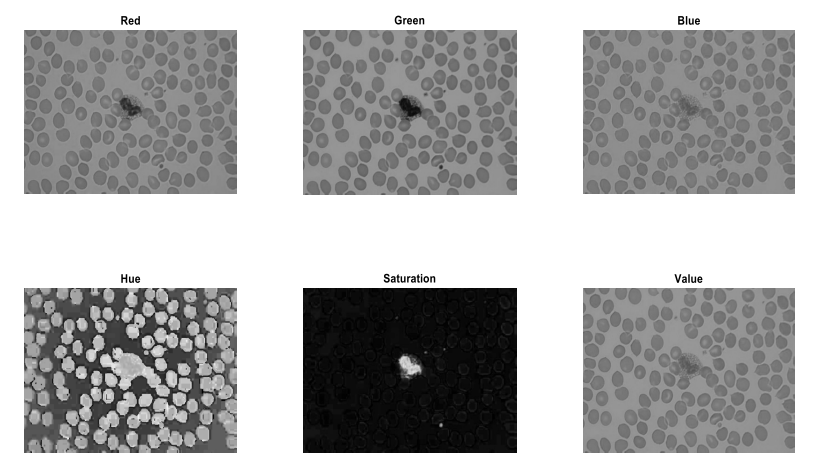

Fig. 4. Microscopic image components in RGB and HSV color spaces.
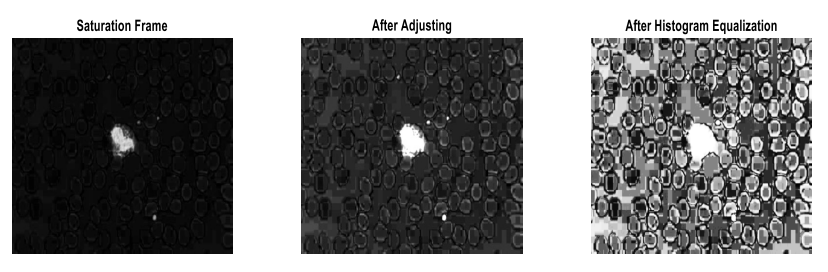

Fig. 5. The input and output images of the image enhancement process.

\subsection{Image Segmentation}

Accurate image segmentation would produce accurate results in the classification process [9]. Therefore, segmentation is the most important and crucial step in the hematological image processing and segmentation systems. In our proposed approach, a simple and quick stepwise thresholding method was used to segment the WBCs. Since saturation frame was chosen in the previous step, WBCs are expected to have the maximum intensity value in the image.

In the thresholding technique, we use the adaptive thresholding technique all image shows a threshold value above 0.95 . This threshold shows only the brightest objects in the image which represent the WBCs as shown in Figure 6 A. After that, the image in hand is subject to a morphological operation to eliminate the undesirable remaining components. It is done by image opening which consists of two main sub-operations, the image erodes followed by image dilations. The operation is applied using disk structure element with a radius of 15 pixels as shown in Figure 6 B. Finally, the bounding box of the region is determined. It represents the largest rectangle containing the segmented WBC area and can be used to crop both the segmented and original WBC image. The final image is a result of multiplying the cropped original and segmented image. It represents the segmented WBC cell in color mode as shown in Figure $6 \mathrm{C}$.

\subsection{Feature Extraction}

Recent research shows that feature extraction of WBC is vital for automated WBC classification. Features provide information that helps to distinguish between various types of WBCs [11]. Different sets of features based on their corresponding target WBC type have been used so far. Such as morphological, discrete cosine transform, and wavelet transform features. In our proposed method, a set of 
morphological, textural, and statistical features were adopted.

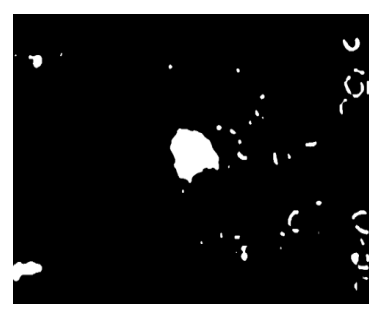

A

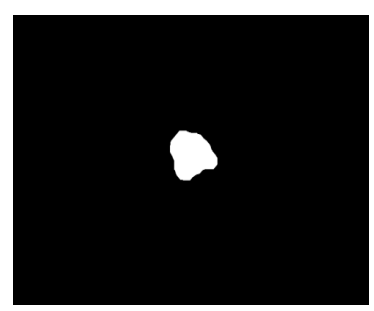

B

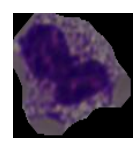

C

Fig. 6. A: The image $5 \mathrm{C}$ after applying thresholding, B: The image after the morphological operation. C: The final segmented WBC from the original image.

\section{A. Morphological Features}

These features deal with the morphology of the extracted WBC image mask. Image mask represents the segmented WBC after eliminating the undesirable remaining components. Two main features were used to distinguish between the cells based on their morphology, equivalent diameter, and circularity. The equivalent diameter is the diameter of the best-fitted circle on the segmented WBC as shown in the equation below.

$$
\text { Equivalent Diameter }=\sqrt[2]{\frac{4 * A}{\pi}}
$$

Where $(A)$ represents the area of the segmented WBC.

The second feature is the object circularity which represents value to determine how much the roundness of the segmented WBC and calculated as shown below.

$$
\text { Circularity }=\frac{P^{2}}{4 * \pi * A}
$$

Where $(P)$ represents the perimeter of the segmented WBC.

\section{B. Textural Features}

Textural features extract information about the spatial arrangement in cropped segmented WBC image. In this paper, the gray level co-occurrence matrices (GLCM) features were used. They include energy, correlation, homogeneity, and contrast. They can be calculated using the following formulas,

$$
\begin{aligned}
& \text { Energy }=\sum_{i=1}^{M} \sum_{j=1}^{N} \operatorname{Im}(i, j)^{2} \\
& \text { Correlation }=\frac{\sum_{i=1}^{M} \sum_{j=1}^{N}(i, j) \operatorname{Im}(i, j)-\mu_{x} \mu_{y}}{\sigma_{x} \sigma_{y}} \\
& \text { Homogeneity }=\sum_{i=1}^{M} \sum_{j=1}^{N} \frac{\operatorname{Im}(i, j)}{1-|i-j|} \\
& \text { Contrast }=\sum_{i=1}^{M} \sum_{j=1}^{N}|i-j|^{2} \operatorname{Im}(i, j)
\end{aligned}
$$

\section{Statistical Features}

The histogram of the cropped segmented WBC image provides information about the distribution of the colors inside the WBC. Statistical analysis of the histogram gives a set of features that includes entropy, skewness, kurtosis, standard deviation and mean. They can be calculated using the following formulas,

$$
\begin{aligned}
& \text { Entropy }=\sum_{i=1}^{M} \sum_{j=1}^{N}-1\left(p_{i, j} * \log _{10}\left(p_{i, j}\right)\right) \\
& \text { Kurtosis }=\sum_{i=1}^{M} \sum_{j=1}^{N}|i-j|^{2} \operatorname{Im}(i, j) \\
& \text { Standard Deviation }=\sqrt{\frac{\sum_{i=1}^{M} \sum_{j=1}^{N}(\operatorname{Im}(i, j)-m e a n)^{2}}{M * N}} \\
& \text { Mean }=\sum_{i=1}^{M} \sum_{j=1}^{N} \frac{\operatorname{Im}(i, j)}{M * N} \\
& \text { Skewness }=\frac{\frac{\sum_{i=1}^{M} \Sigma_{j=1}^{N}(\operatorname{Im}(i, j)-\mu)^{3}}{M * N}}{\left(\sqrt{\frac{\sum_{i=1}^{M} \Sigma_{j=1}^{N}(\operatorname{Im}(i, j)-\mu)^{2}}{M * N}}\right)^{3}}
\end{aligned}
$$

\section{Principle Component Analysis}

Since some of the features may not be of many effects on the accuracy of classifiers, the Principle Component Analysis (PCA) procedure can be used to reduce the dimensions of the extracted features vector. Consequently, reducing the required time of the process of segmentation till classification. The extracted features vectors are fed into the PCA which uses the correlation matrix instead of the covariance matrix since different units occur in the feature set [24]. After applying this procedure, the eigenvalues and variances of inputs are calculated. Also, the set of main components is obtained and arranged based on their ability to distinguish between the different types of WBCs.

\section{Classification}

After segmentation of each WBCs and extracting their features, It is required to classify the segmented images into five types of WBCs (basophil, eosinophil, monocyte, lymphocyte, and neutrophil) using pattern recognition techniques. Three different types of classifiers were exploited which are a probabilistic neural network (PNN), support vector machine (SVM), and RandomForest.

PNN is a feedforward neural network classifier where the probability distribution function (PDF) of each layer is approximated. This is done by using parzen window and non-parametric function which is required in Bayesian rule step [25]. It consists of four main parts, the input, pattern, summation, and output which are fully interconnected. The pattern part is activated using an exponential function instead of a sigmoidal which is usually used in other types of neural networks. The exponential function computes the distances from the input vector to the training input vectors. For each new input vector, the PNN algorithm produces a vector that provides an indication of how close the input is to a training input. The summation part will sum the contributions of each input class and produces a single output, which is called a vector of probabilities. The class with the highest probability is selected as the class type.

$\mathrm{SVM}$ is one of the most common classification algorithms which is used in the medical field. The basic concept of SVM is the decision planes which are used to define decision boundaries. Decision planes are used to separate the features to different classes and the simplest plane is the linear one [23]. In our proposed method, the features set are 
not linearly separable. Hence, kernel SVM with fifth order polynomial function was used.

Random forests (RF) classifier, first proposed by Breiman [26], is one of the most popular classification tools and excellent ensemble machine learning techniques. The main idea of RF classifier is to build classification trees based on some randomly selected features from randomly selected samples with bagging strategy. Then to use the built trees to vote for a given input vector to get a class label. RF classifiers are constructed by many base learners and each one is an independent binary tree adopting recursive partitioning. RF has many advantages as follows: provides higher accuracy than other classifiers, efficient on largescale data, does not overfit, and can be easily applied in multi-class inputs.

\section{Results and Discussion}

To validate the proposed method in this paper, two different datasets were used to approve the segmentation process and ensure that the method covers varies types of images and cell situation. The first dataset is LISC database that was presented by Rezatofighi et al., a 400 samples images were taken from 100 microscope slides, images were acquired by a light Microscope-Axioskope 40 using an achromatic lens with a magnification of 100 [5].

The second dataset was collected by Sarrafzadeh et al., the dataset is prepared with Medical Image and Signal Processing Research Center (MISP) and Department of Pathology at Isfahan University of Medical Sciences. The dataset contains 100 microscopic images of blood smears with ECLIPSE 50i microscope of a magnification of 100 $[10,11]$. Both datasets, MISP and LISC, were used to evaluate the segmentation algorithm while the latter is used to evaluate the classification process.

This section is to study the performance of the proposed segmentation method. The accuracy of each WBC type and the overall accuracy are calculated and shown in table 2 . In our experiment, 594 blood smear images are used, and the proposed method shows high accuracy in segmenting of the five white blood cells with an overall accuracy of $97.94 \%$. Figure 7 shows an example of the segmented cells using the proposed algorithm.

Table 2. Detection accuracy of five types of white blood cells using the proposed method compared with other methods on literature.

\begin{tabular}{c|c|c|c|c}
\hline \multirow{2}{*}{ Type } & \multicolumn{4}{|c}{ Accuracy \% } \\
\cline { 2 - 5 } & $\begin{array}{c}\text { Proposed } \\
\text { Method }\end{array}$ & {$[\mathbf{5 ]}$} & {$[\mathbf{2 2}]$} & {$[\mathbf{2 3}]$} \\
\hline Basophil & 100 & 94.70 & 100 & 89.70 \\
Lymphocyte & 91.52 & 90.01 & 92.30 & 97.00 \\
Monocyte & 100 & 91.23 & 92.30 & 97.80 \\
Eosinophil & 99.50 & 93.22 & 96.15 & 89.40 \\
Neutrophil & 98.70 & 96.23 & 96.15 & 97.60 \\
Average \% & 97.94 & 93.09 & 95.38 & 94.30 \\
\hline
\end{tabular}

\begin{tabular}{ccc}
$\begin{array}{c}\text { Cropped Original } \\
\text { Image Based in } \\
\text { Segmentation }\end{array}$ & $\begin{array}{c}\text { Segmentation } \\
\text { Mask }\end{array}$ & $\begin{array}{c}\text { Masked } \\
\text { Original Image }\end{array}$ \\
\hline
\end{tabular}
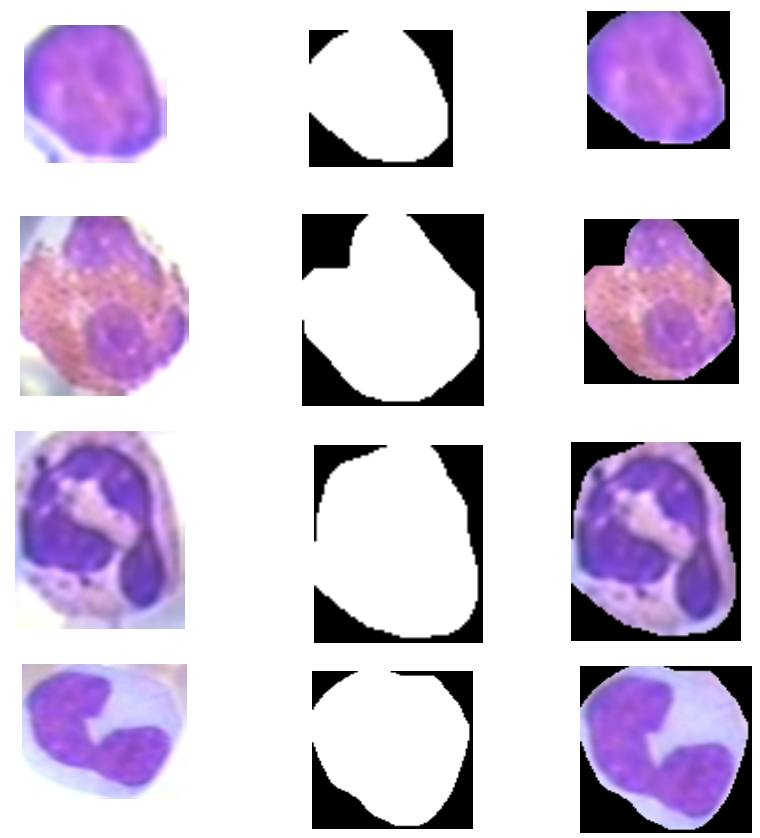

Fig. 7. Example of segmented cells using the proposed method.

After successfully segmenting the WBCs with high accuracy, features vector is extracted and fed into the PCA to be ordered based on their contribution on discrimination between different types of WBCs. The classifiers performance was studied for 4, 8, and 11 features based on their ordering output of PCA. It is important to note that the maximum number of features that were considered is only 11 features.

The most important parameter that influences the classification process in the PNN classifier is the spread constant. In order for it to be determined, the variation of classifier output of 11 features among the spread constant was studied. Figure 8 shows the variation of accuracy over spread constant value. The results are based on that the optimum value of spread function that was chosen of value 0.2 .

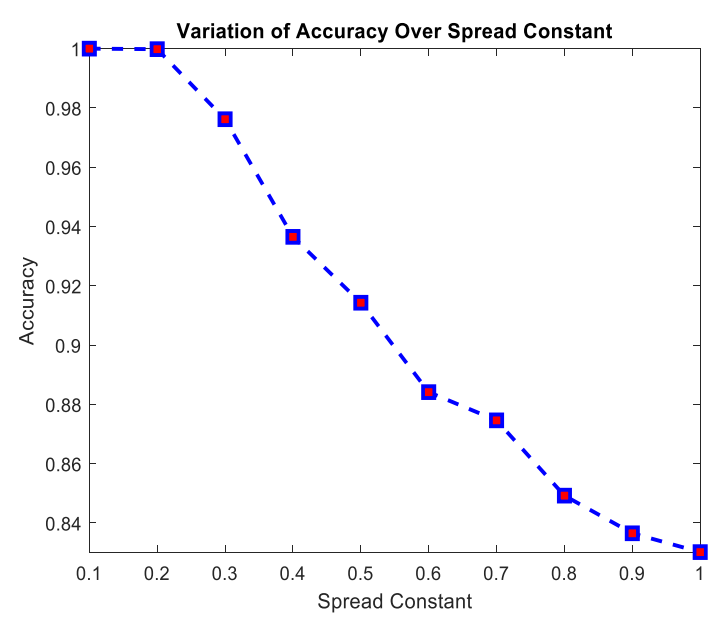

Fig. 8. Accuracy variation over spread constant for PNN.

In the next step, a vector of 4,8 or 11 features was fed to the three types of classifiers PNN, SVM, and Random Forest. The order of the features is based on the one generated by PCA in the previous step. The resulting features dataset is then used to determine the subtype of the segmented WBC using the three types of classifiers. The accuracy, specificity, and sensitivity of the used classifier 
and their changes over the number of principal components used are shown in Figure 9, Figure 10, and Figure 11 respectively.

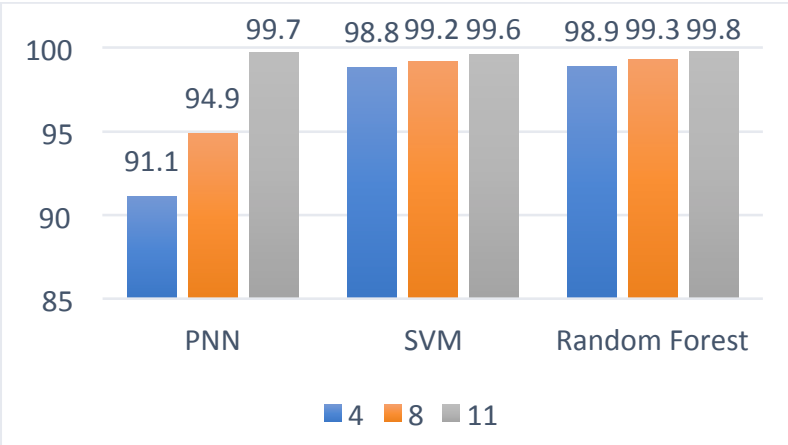

Fig. 9. Accuracy variation over a number of used features $(4,8,11)$.

As shown in Figure 9 above random forest classifier is the most stable classifier regarding sensitivity change over the used number of features then SVM and finally PNN.

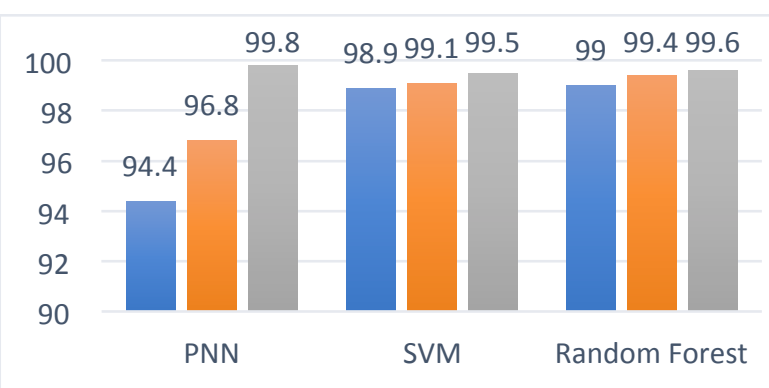

$\square \quad \square \quad 11$

Fig. 10. Sensitivity variation over a number of used features $(4,8,11)$.

Figure 10 above shows that random forest classifier is the most stable classifier regarding sensitivity variation over the used number of features then SVM and finally PNN.

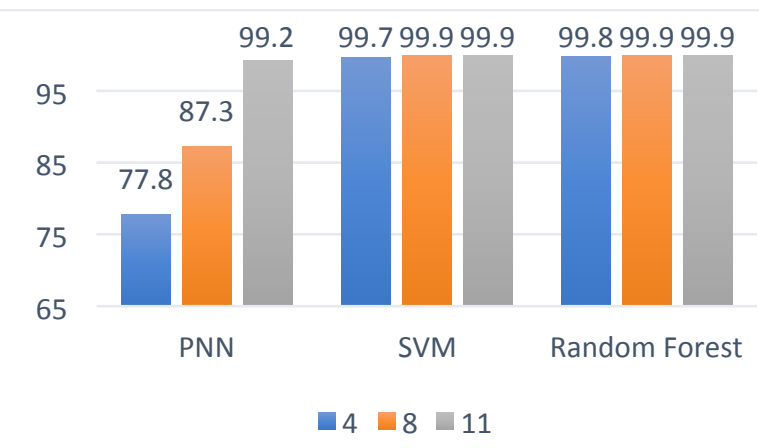

Fig. 11. Specificity variation over a number of used features $(4,8,11)$.

As shown in Figure 11 above random forest classifier is the most stable classifier regarding specificity change over the used number of features then SVM and finally PNN. Based on these results we can conclude that random forest and SVM classifiers are more stable regarding a number of used features than the PNN classifier and has a higher performance than PNN.

It is very important to note that the results in the figure above show that our proposed method provides high accuracy even in a small number of principal components. For two type of classifiers SVM and Random Forest, they achieve $98.8 \%$, and $98.9 \%$ respectively with principal components of value 4 . In addition, we can add that by increasing the number of principal components all classifiers achieve very high accuracy (PNN: 99.8\%, SVM: 99.5\%, and Random Forest: 99.6\%). Moreover, the use of accurate segmentation methodology and highly descriptive features (Morphological, Textural, and Statistical) of the cells allowing the classification process to easily identify and classify WBCs. Table 3 shows a comparison for classification accuracy between proposed method and methods in the literature.

Table 3. Classification accuracy of WBCs using proposed method compared to methods in the literature.

\begin{tabular}{c|c|c|c}
\hline & $\begin{array}{c}\text { Accurac } \\
\mathbf{y} \%\end{array}$ & $\begin{array}{c}\text { Sensitivity } \\
\mathbf{\%}\end{array}$ & $\begin{array}{c}\text { Specificity } \\
\mathbf{\%}\end{array}$ \\
\hline $\begin{array}{c}\text { Proposed } \\
\text { Method } \\
\text { PNN }\end{array}$ & 99.70 & 99.2 & 99.8 \\
\hline $\begin{array}{c}\text { Proposed } \\
\text { Method } \\
\text { SVM }\end{array}$ & 99.60 & 99.9 & 99.5 \\
\hline $\begin{array}{c}\text { Proposed } \\
\text { Method } \\
\text { RF }\end{array}$ & 99.80 & 99.9 & 99.6 \\
\hline$[5]$ & 93.00 & 96.97 & 94.74 \\
\hline [7] & 97.3 & 96.00 & 98.80 \\
\hline$[11]$ & 97.73 & 98.25 & 95.95 \\
\hline
\end{tabular}

In this part, we analyze the computational time of the proposed method to highlight its computation feature. For this purpose, images are used in full and passed through the proposed method. WBCs segmentation and classification for three different number of features. Preprocessing and the Segmentation process time is constant since it's don't depend on the number of features while extracting an 11 set of features, classifier training, and testing consumes more time than 4 and 8 features. Table 4 shows full details about time consumption for each computational step in the proposed algorithm. The time consumption is calculated over the variation of a number of used features selected using PCA.

Table 4. Approximate Time consumption (in Seconds) for a different number of features $(4,8$, and 11$)$ in the different computational stage.

\begin{tabular}{c|c|c|c}
\hline \multirow{2}{*}{$\begin{array}{c}\text { Computational stage of } \\
\text { the proposed system }\end{array}$} & \multicolumn{3}{|c}{ Number of Features } \\
\cline { 2 - 4 } & $\mathbf{3}$ & $\mathbf{3}$ & $\mathbf{8}$ \\
\hline Time (sec) \\
Pre-processing & 3.467 & 3.467 & 3.467 \\
Segmentation & 1.329 & 1.329 & 1.329 \\
Features extraction & 1.534 & 3.712 & 8.134 \\
Classifier training & 3.412 & 6.532 & 8.315 \\
Testing & 1.272 & 1.813 & 2.376 \\
Total & 11.0140 & 16.8530 & 23.6210 \\
\hline
\end{tabular}

\section{Conclusion}

In this paper, a new fully automated white blood cells segmentation and classification based on the color space transformation and morphological methods are proposed. The uses of saturation frame in HSV color space are utilized and an image adjustment and histogram equalization are applied to enhance the contrast between the WBC and other blood components. Then, a simple thresholding technique is 
performed to extract the brightest objects of the image. Finally, the morphological operation is applied to remove unwanted components. The overall accuracy of the segmentation process is $97.94 \%$ for all types of WBCs.

For feature extraction and classification of WBCs, the paper combines the morphological features, textural features, and statistical features to produce features set vector. It is important to mention that the maximum number of extracted features used is 11 which would reflect the time required by the classifiers. The features set are ordered by the PCA to select the feature with the highest contribution to discrimination between different types of WBCs. Finally, a classification process is applied to the features set and we have obtained $99.7 \%, 99.6 \%, 99.8 \%$ accuracy for PNN, SVM and Random Forest classifiers respectively.

This is an Open Access article distributed under the terms of the Creative Commons Attribution Licence

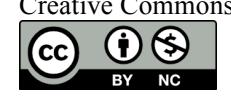

\section{References}

[1] Ghane, N., Vard, A., Talebi, A. and Nematollahy, P., 2017. Segmentation of white blood cells from microscopic images using a novel combination of K-means clustering and modified watershed algorithm. Journal of medical signals and sensors, 7(2), p.92. PMID: 28553582

[2] Sajjad, M., Khan, S., Shoaib, M., Ali, H., Jan, Z., Muhammad, K. and Mehmood, I., 2016, December. Computer aided system for leukocytes classification and segmentation in blood smear images. In Frontiers of Information Technology (FIT), 2016 International Conference on (pp. 99-104). IEEE. DOI: 10.1109/FIT.2016.026

[3] Sural, S., Qian, G. and Pramanik, S., 2002. Segmentation and histogram generation using the HSV color space for image retrieval. In Image Processing. 2002. Proceedings. 2002 International Conference on (Vol. 2, pp. II-II). IEEE. DOI: 10.1109/ICIP.2002.1040019

[4] Siqiang, L. and Wei, L., 2007, July. Image segmentation based on the Mean-Shift in the HSV space. In Control Conference, 2007. CCC 2007. Chinese (pp. 476-479). IEEE.DOI:10.1109/CHICC.2006.4346838

[5] Rezatofighi, S.H. and Soltanian-Zadeh, H., 2011. Automatic recognition of five types of white blood cells in peripheral blood. Computerized Medical Imaging and Graphics, 35(4), pp.333-343.DOI:10.1016/j.compmedimag.2011.01.003

[6] Umpon NT, Dhompongsa S. Morphological granulometric features of nucleus in automatic bone marrow white blood cell classification. IEEE Trans Inf Technol Biomed 2007; 11:353-9. DOI: 10.1109/TITB.2007.892694

[7] Prinyakupt, J. and Pluempitiwiriyawej, C., 2015. Segmentation of white blood cells and comparison of cell morphology by linear and naïve Bayes classifiers. Biomedical engineering online, 14(1), p.63.DOI: 10.1186/s12938-015-0037-1

[8] Scotti, F., 2006, April. Robust segmentation and measurements techniques of white cells in blood microscope images. In Instrumentation and Measurement Technology Conference, 2006. IMTC 2006. Proceedings of the IEEE (pp. 43-48). IEEE.DOI:10.1109/IMTC.2006.328170

[9] ElDahshan, K.A., Youssef, M.I., Masameer, E.H. and Hassan, M.A., 2015. Comparison of segmentation framework on digital microscope images for acute lymphoblastic leukemia diagnosis using RGB and HSV color spaces. Journal of Biomedical Engineering and Medical Imaging, 2(2), p.26.DOI: 10.14738/jbemi.22.1065

[10] Sarrafzadeh, O., Dehnavi, A.M., Rabbani, H., Ghane, N. and Talebi, A., 2015, October. Circlet based framework for red blood cells segmentation and counting. In Signal Processing Systems (SiPS), 2015 IEEE Workshop on (pp. 1-6). IEEE.DOI:10.1109/SiPS.2015.7344979

[11] Sarrafzadeh, O., Rabbani, H., Talebi, A. and Banaem, H.U., 2014. Selection of the best features for leukocytes classification in blood smear microscopic images. SPIE Medical Imaging. International Society for Optics and Photonics, 90410.DOI:10.1117/12.2043605

[12] Strzelecki, M., Szczypinski, P., Materka, A. and Klepaczko, A., 2013. A software tool for automatic classification and segmentation of 2D/3D medical images. Nuclear Instruments and Methods in Physics Research Section A: Accelerators, Spectrometers, Detectors and Associated Equipment, 702, pp.137140.DOI:10.1016/j.nima.2012.09.006
[13] Reta, C., Gonzalez, J.A., Diaz, R. and Guichard, J.S., 2011. Leukocytes segmentation using Markov random fields. In Software Tools and Algorithms for Biological Systems (pp. 345-353). Springer New York.DOI: 10.1007/978-1-4419-70466_35

[14] Ravikumar, S., 2016. Image segmentation and classification of white blood cells with the extreme learning machine and the fast relevance vector machine. Artificial cells, nanomedicine, and biotechnology, 44(3), 989.DOI:10.3109/21691401.2015.1008506

[15] Huang, D.C., Hung, K.D. and Chan, Y.K., 2012. A computer assisted method for leukocyte nucleus segmentation and recognition in blood smear images. Journal of Systems and Software, 85(9), pp.2104-2118.DOI:10.1016/j.jss.2012.04.012

[16] Li, Y., Zhu, R., Mi, L., Cao, Y. and Yao, D., 2016. Segmentation of white blood cell from acute Lymphoblastic Leukemia images using dual-threshold method. Computational and mathematical methods in medicine, 2016.DOI: 10.1155/2016/9514707

[17] Chinnathambi, K., Ramasamy, A. and Ramachandran, P., 2014. Robust segmentation of cancer affected white blood cells using modified level set algorithm. International Journal of Simulation: Systems, $\quad$ Science
Technology, 14.DOI:10.5013/IJSSST.a.14.01.02

[18] Chan, Y.K., Tsai, M.H., Huang, D.C., Zheng, Z.H. and Hung, K.D., 2010. Leukocyte nucleus segmentation and nucleus lobe counting. BMC bioinformatics, 11(1), p.558.DOI: 10.1186/14712105-11-558

[19] Liu, Z., Liu, J., Xiao, X., Yuan, H., Li, X., Chang, J. and Zheng, C., 2015. Segmentation of white blood cells through nucleus mark watershed operations and mean shift clustering. sensors, 15(9), pp.22561-22586.DOI: 10.3390/s150922561

[20] Ramesh, N., Dangott, B., Salama, M.E. and Tasdizen, T., 2012. Isolation and two-step classification of normal white blood cells in peripheral blood smears. Journal of pathology informatics, 3.DOI: 10.4103/2153-3539.93895

[21] Gautam, A. and Bhadauria, H., 2014, September. Classification of white blood cells based on morphological features. In Advances in Computing, Communications and Informatics (ICACCI, 2014 International Conference on (pp. 2363-2368). IEEE.DOI:10.1109/ICACCI.2014.6968362

[22] Shirazi, S.H., Umar, A.I., Naz, S. and Razzak, M.I., 2016. Efficient leukocyte segmentation and recognition in peripheral blood image. Technology and Health Care, 24(3), pp.335-347. DOI:10.3233/THC-161133

[23] Sajjad, M., Khan, S., Jan, Z., Muhammad, K., Moon, H., Kwak, J.T., Rho, S., Baik, S.W. and Mehmood, I., 2017. Leukocytes classification and segmentation in microscopic blood smear: a resource-aware healthcare service in smart cities. IEEE Access, 5, pp.3475-3489. DOI:10.1109/ACCESS.2016.2636218

[24] Alqudah, A.M., Algharib, H.M., Algharib, A.M. and Algharib, H.M., 2018. COMPUTER AIDED DIAGNOSIS SYSTEM FOR AUTOMATIC TWO STAGES CLASSIFICATION OF BREAST MASS IN DIGITAL MAMMOGRAM IMAGES. Biomedical Engineering: Applications, Basis and Communications, p.1950007. DOI: $10.4015 / \mathrm{S} 1016237219500078$

[25] D. Specht, "Probabilistic neural networks", Neural Networks, vol. 3, no. 1, pp. 109-118, 1990. DOI:10.1016/0893-6080(90)90049-Q

[26] L. Breiman, Machine Learning, vol. 45, no. 1, pp. 5-32, 2001. DOI: 10.1023/A:1010933404324 